\title{
Photonics Improvement of the Time-Bandwidth Product for a Linearly Chirped Waveform
}

\author{
Xuan Li, Shanghong Zhao, Guodong Wang, He Li \\ Information and Navigation College, Air Force Engineering University, Xi'an, China \\ Email: lixuanrch@163.com
}

How to cite this paper: Li, X., Zhao, S.H., Wang, G.D. and Li, H. (2020) Photonics Improvement of the Time-Bandwidth Product for a Linearly Chirped Waveform. Journal of Applied Mathematics and Physics, 8, 2955-2963.

https://doi.org/10.4236/jamp.2020.812218

Received: July 21, 2020

Accepted: December 19, 2020

Published: December 22, 2020

\begin{abstract}
A photonics approach to generate a linearly chirped waveform with increased TBWP is proposed and investigated. The time bandwidth product (TBWP) of the linearly chirped waveform is improved based on optical microwave frequency multiplying combined with temporal synthesis. An integrated dual-polarization modulator and an optical filter are utilized to perform frequency doubling operation by generating an orthogonally polarized optical signal, which consists of an optical carrier in one polarization direction and a second-order chirped optical sideband in another. Then the orthogonally polarized optical signal puts into a polarization modulator (PolM) to perform phase coding process. By driving a Pseudorandom (PN) sequence to the PolM, the time duration of the generated bandwidth doubled linearly chirped waveform can be synthesized to arbitrary length. The approach is verified by simulation. A linearly chirped waveform with central frequency of $8.25 \mathrm{GHz}$, bandwidth of $500 \mathrm{MHz}$, time duration of $6.4 \mathrm{~ns}$ is used to generate a synthesized waveform with central frequency of $16.5 \mathrm{GHz}$, bandwidth of $1 \mathrm{GHz}$, time duration of 819.2 ns. The TBWP of the linearly chirped signal is improved from 3.2 to 819.2. The proposed method features arbitrary large TBWP, and it can be used in a radar system to improve its resolution.
\end{abstract}

\section{Keywords}

Linearly Chirped Waveform, Time-Bandwidth Product, Frequency Multiplying, Phase Coding

\section{Introduction}

Modern radar systems require large time-bandwidth product (TBWP) in order to simultaneously improve location and velocity measurement accuracies of a 
target. Linearly chirped signal is a widely used radar waveform to obtain high measurement resolution through pulse compression [1]. Nevertheless, a chirped waveform which is generated based on electronics technique suffers from electrical bottlenecks, such as limited digital-to-analog conversion speed, high timing jitter, sensitive electromagnetic interference, high loss and large size. Compared to electronics one, photonics technique is fundamentally capable of generating, delivering and processing microwave signal with broader bandwidths, higher frequency, larger tunable range and greater flexibility [2] [3].

Different photonics techniques have been demonstrated to generate linearly chirped waveforms, including direct space-to-time mapping [4], spectral shaping combined with frequency-to-time mapping (FTTM) [5], interference of two ultra-short optical pulses with different dispersions [6] [7], phase modulating two continuous optical wavelengths with an electrical parabolic waveform [8], and so on. However, the time-bandwidth produce (TBWP) of the generated chirped waveforms based on the mentioned schemes still limited. For example, the first three methods will generate linearly chirped signals with very limited temporal pulse duration, while the fourth method will generate waveforms with limited bandwidth. Therefore, the TBWP of the generated signal should be improved by using photonics technique. The most widely used method to improve the TBWP of a linearly chirped signal is optical microwave frequency multiplying. However, when a large multiplying factor is achieved, the configuration will be complicated and the signal-to-noise ratio (SNR) of the generated waveform will be seriously deteriorated due to the finite extinction ratio of the modulator and the frequency multiplying operation. Recently, high-speed phase and amplitude modulation is inserted in the spectral shaping and FTM scheme to synthesize a linearly chirped waveform with long time duration and large TBWP [9] [10] [11], but the system is complicated and the stability is poor due to the use of spatially separated structure. Phase coding also employed to improve the TBWP of a linearly chirped signal by driving a phase-coded parabolic waveform to a polarization modulator (PolM) [12], however, the electrical signal is very complicated, which may hinder the application.

In this paper, we propose a photonics approach to improve the TBWP of a linearly chirped waveform based on optical microwave frequency multiplying combined with temporal synthesis. As the frequency multiplying factor equals 2 , the SNR will be slightly deteriorated (theoretically decreased by $6 \mathrm{~dB}$ ). While for the temporal synthesis operation, the phase coding will be performed with the help of polarization modulation, as the spatial separate process is avoided, the stability will be guaranteed.

\section{Principle}

Figure 1 shows the schematic configuration of the proposed TBWP improvement system, which consists of a laserdiode (LD), an integrated dual-polarization quadrature phase shift keying (DP-QPSK) modulator, an optical filter, a PolM, a 


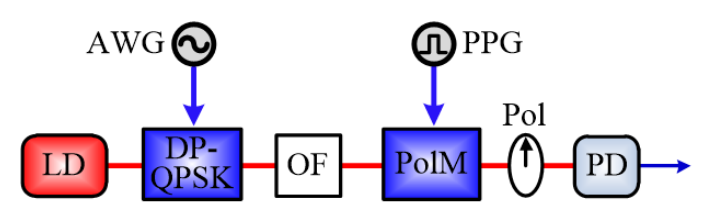

Figure 1. Schematic configuration of the proposed linearly chirped waveform generator with improved TBWP. LD, laserdiode; DP-QPSK, dual-polarization quadrature phase shift keying; AWG, arbitrary waveform generator; OF, optical filter; PolM, polarization modulator; PPG, pulse pattern generator; Pol, polarizer; PD, photodetector.

polarizer (Pol) and a photodetector (PD). The lightwave from the $\mathrm{LD}$ is sent into the integrated modulator. The DP-QPSK modulator consists of a 3-dB optical coupler, two QPSK modulators and a polarization beam combiner (PBC), and each of the QPSK modulators consists of two sub-MZMs placed parallel in a main-MZM. The integrated device has four RF input ports, ports 1 and 2 correspond to the upper QPSK modulator while ports 3 and 4 correspond to the bottom one. In our scheme, only port 1 is driven by an electrical linearly chirped signal. At the output of the integrated modulator, the optical signals from the two QPSK modulators are combined with orthogonal polarization states through the PBC.

When only the RF input port 1 is connected to the driving linearly chirped signal, a frequency and bandwidth doubled chirped waveform can be generated, as shown in Figure 2. Figure 2(a) shows the optical signals in the DP-QPSK modulator. In the upper QPSK modulator, one sub-MZM is driven by the chirped signal to generate optical carrier and second-order sidebands. By adjusting the DC bias voltages of the other sub-MZM and the main-MZM to make the two optical carriers from the two sub-MZMs have identical powers and opposite phases, then the optical carriers canceled each other and only the second-order sidebands obtained. While for the bottom QPSK modulator, the optical carrier is simply passed through. At the output of the DP-QPSK modulator, the signals from the two QPSK modulators are combined with orthogonal polarization states, as shown in Figure 2(b). Then, a polarization-independent optical filter is followed by to filter the optical carrier and one chirped sideband (e.g. lower second-order sideband). We have investigated this modulation scheme in details in our previous work [13].

At the output of the OF, the optical signal can be given by

$$
\left[\begin{array}{c}
E_{x}(t) \\
E_{y}(t)
\end{array}\right] \propto e^{j \omega_{c} t}\left[\begin{array}{c}
A_{2} e^{-j 2\left(\omega_{1} t-k t^{2}\right)} \\
A_{0}
\end{array}\right], 0 \leq t \leq T
$$

where $x$ and $y$ represent the principal axes of the PBC, $A_{0}$ and $A_{2}$ represent the amplitude of the carrier and sideband components, respectively, $\omega_{1}$ and $k$ are the initial angular frequency and chirp rate of the driving signal which provided by an arbitrary waveform generator (AWG), $T$ is the time duration of the driving signal.

Then, the optical signal puts into the PolM to perform phase coding process, 

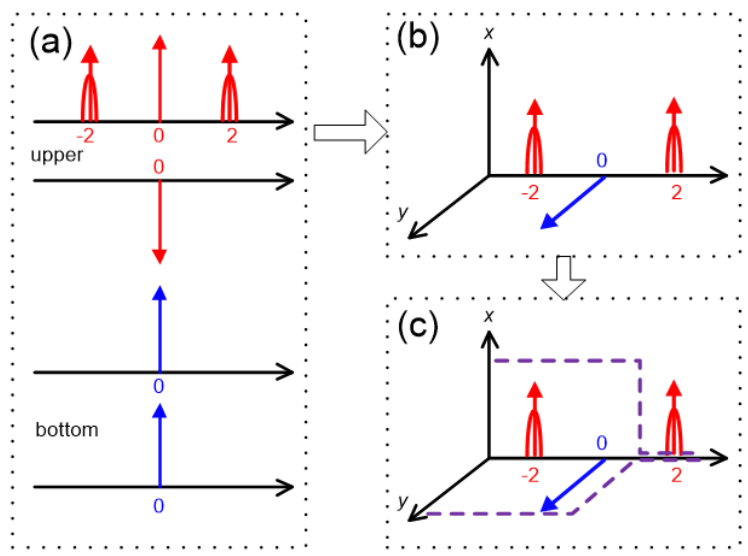

Figure 2. The optical spectra and its polarization states of the optical signals (a) in DP-QPSK modulator, (b) after DP-QPSK modulator, (c) in OF.

the Pol is followed by to project the two orthogonal components in the same direction, and the output of the Pol can be given by

$$
E_{p o l}(t) \propto A_{2} e^{j \omega_{c} t} e^{-j 2\left(\omega_{1} t-k t^{2}\right)} e^{-j m s[i]}+A_{0} e^{j \omega_{c} t} e^{j m s[i]}, 0 \leq t \leq T
$$

where $m$ is the modulation index of the PolM, and $s[i]$ is the element of the binary sequence.

Finally, the optical signal puts into the PD, a phase-coded frequency and bandwidth doubled linearly chirped signal can be obtained as

$$
i(t) \propto A_{0} A_{2} \cos \left[2 \omega_{1} t-2 k t^{2}+2 m s[i]\right], 0 \leq t \leq T
$$

To optimal the phase coding performance, the modulation index $m$ should be set as $\pi / 2$ when $s[i]$ has value of " 0 " or " 1 ". Then the equation can be expressed as

$$
i(t) \propto(-1)^{s[i]} \cos \left(2 \omega_{1} t-2 k t^{2}\right), 0 \leq t \leq T
$$

Assuming a single linearly chirped waveform $w(t)$ has an auto-correlation function of $R_{s}(\tau)$, which can be expressed as

$$
R_{s}(\tau)=\int w(t) w(t-\tau) d t
$$

The sequence $s[i]$ has a length of $L$, the auto-correlation function of the discrete term $(-1)^{s[i]}$ can be expressed as

$$
R_{s}(n)=\sum_{m=0}^{L-1}(-1)^{s[m]} \cdot(-1)^{s[n-m]}
$$

When the binary sequence is set as Pseudorandom (PN) sequence, due to the properties of the PN sequence, the following condition will be satisfied [14]

$$
R_{s}(n)=\left\{\begin{array}{l}
L n=0, \pm L, \pm 2 L, \ldots \\
-1 \text { elsewhere }
\end{array}\right.
$$

The auto-correlation function of PN sequence have maximum value of $L$ and repeating period of $L$. As a result, when $L>>1$, the auto-correlation function of the generated phase-coded chirped signal can be written as 


$$
R(\tau)=\sum_{i} L R_{s}(\tau) * \delta(t-i L T)
$$

As can be seen, the main peaks of auto-correlation have a repetitive period of $T L$, indicating that by phase coding the repetitive basis linearly chirped train with PN sequence, the time duration of the waveform can be synthesized by a factor of $L$.

\section{Simulation Results and Discussion}

A simulation is performed to verify the proposed TBWP improvement scheme. In the simulation, the linearly polarized optical carrier has a central frequency of 193.1 THz. In the upper QPSK modulator, only one sub-MZM is driven by the linearly chirped signal, while another sub-MZM and the main MZM are DC biased to generate two second-order sidebands with the optical carrier suppressed. The driving linearly chirped waveform has a time duration of $6.4 \mathrm{~ns}$ and a repetitive period of $6.4 \mathrm{~ns}$. A pseudo-random bit sequence with data rate of 156.25 Mbit/s is applied to the PolM to modulate the phase of the generated chirped waveform. The modulation indices of the PMs are set as $\pi / 2$.

First, the initial frequency and bandwidth of the driving chirped signal are set as $8.5 \mathrm{GHz}$ and $500 \mathrm{MHz}$, the spectrum of the driving repetitive chirped waveform train is shown in Figure 3(a), as can be seen, the input chirped signal has a central frequency of $8.25 \mathrm{GHz}$ and a bandwidth of $500 \mathrm{MHz}$. Figure 3(b) shows the output spectrum of the PD, the generated signal has a central frequency of 16.5 GHz and a bandwidth of $1 \mathrm{GHz}$. After the PD, a bandpass filter (BPF) is used to filter the useful component form the spectrum. The output waveform of the BPF is shown in Figure 3(c), while the simultaneous frequency of the waveform is extracted by using Hilbert transformation and shown in Figure 3(d).
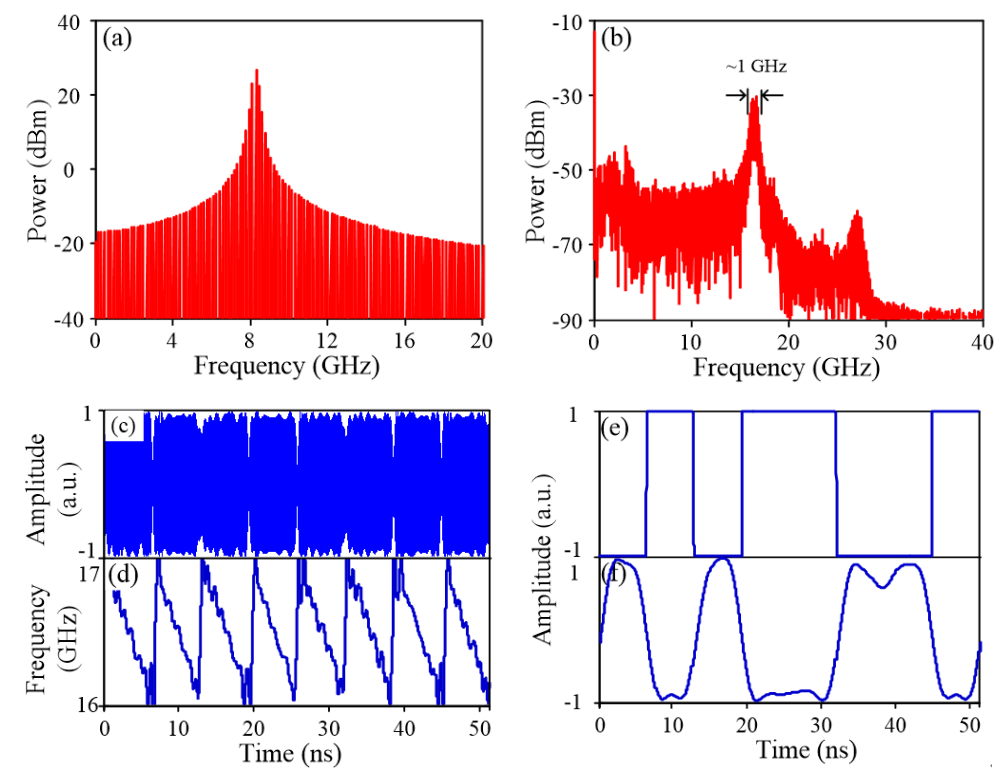

Figure 3. (a) Spectrum of input chirped waveform train, (b) output spectrum of PD, (c) output waveform of BPF, (d) simultaneous frequency extracted from the generated waveform, (e) input binary sequence and (f) demodulated baseband waveform. 
The phase information cannot be directly observed, therefore, we designed a coherent detection system to verify the phase coding performance [15]. At the output of the coherent detection system, a low frequency demodulated waveform is obtained, as shown in Figure 3(f), while Figure 3(e) shows the input binary sequence. As can be seen, the phase coding process is successfully achieved to the linearly chirped waveform train.

The TBWP improvement of the linearly chirped waveform is investigated. For the driving linearly chirped waveform, the TBWP is calculated as $3.2(6.4 \times 0.5)$, for the generated bandwidth doubled basis waveform, the TBWP is calculated as $6.4(6.4 \times 1)$. Figure $4(a)$ shows the auto-correlation function of the generated bandwidth doubled basis waveform, as can be seen, the peak-to-sidelobe ratio (PSR) is $9.1 \mathrm{~dB}$ and the full width at half maximum (FWHM) of the compressed pulse is $1.1 \mathrm{~ns}$. Thus, a pulse compression ratio (PCR) of 5.8 is achieved. To further improve the TBWP of the linearly chirped waveform, a PN sequence with length of 128 is applied to the PolM to modulate the phase of each basis waveform. At a result, a phase-coded bandwidth doubled linearly chirped signal consists of 128 basis waveforms is obtained. The phase-coded signal has a time duration of 819.2 ns. Figure 4(b) shows the auto-correlation function of 128 repetitive basis waveform train without phase coding, while Figure 4(c) shows the auto-correlation function of the generated phase coded linearly chirped signal. As can be seen, due to the phase coding operation, the pulse compression performance is extremely strengthened. The phase-coded bandwidth doubled linearly chirped signal has a PSR of $10.8 \mathrm{~dB}$, at the same time, compared to the basis bandwidth doubled waveform, the FWHM of the phase-coded signal keeps unchanged (1.1 ns), making the PCR increase by 128 times (744.8). The phase-coded
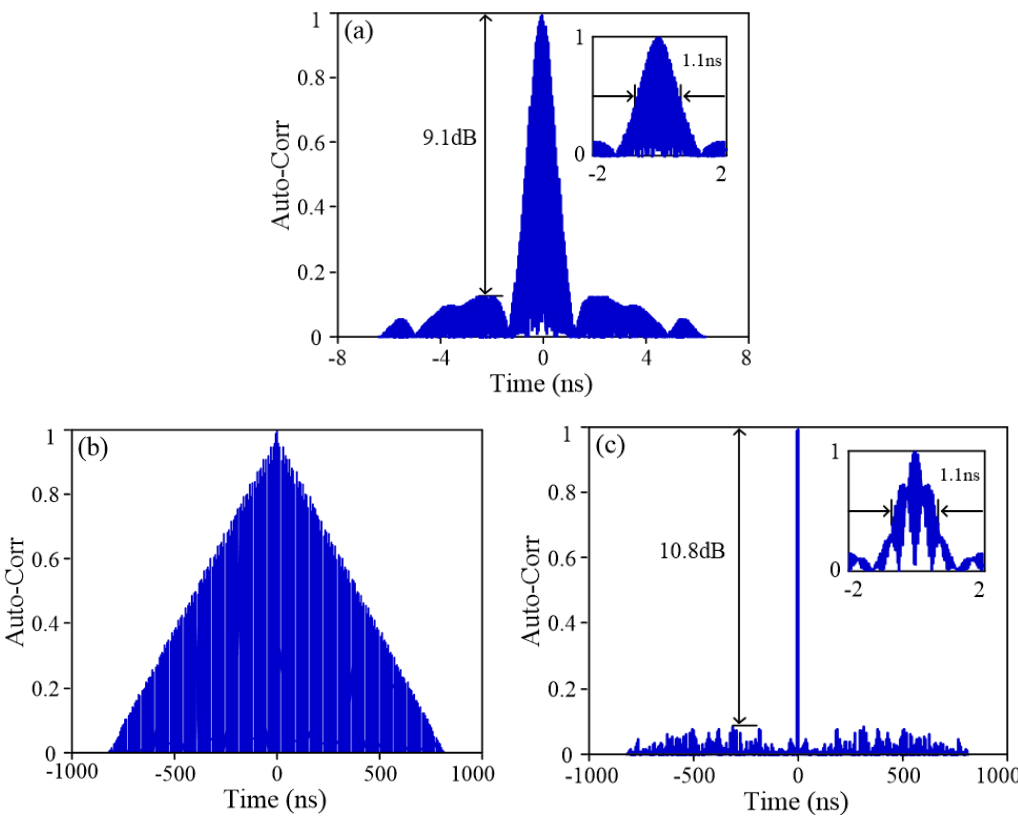

Figure 4. Auto-correlation function of (a) single bandwidth doubled basis waveform, (b) 128 repetitive basis waveforms (c) generated phase-coded linearly chirped signal. 
signal which consists of 128 basis waveforms can be considered as one synthesized waveform to perform target detection. The bandwidth of the synthesized waveform equals the bandwidth of the basis waveform, while the time duration of the synthesized waveform is equal to the time duration of basis waveform times the length of PN sequence. As a result, by temporal synthesis process the TBWP of the bandwidth doubled signal will be further improved to 819.2.

One problem associated with the proposed scheme is the polarization error, i.e. polarization extinction ratio (PER) existed in the phase coding process. We compared two basis waveforms with different modulated phases (i.e. time window is 12.8 ns and corresponding $s[i]$ are " 0 " and " 1 " respectively) when the PER changed from infinite to $9 \mathrm{~dB}$, as shown in Figure 5. As can be seen, when the PER decreased, an amplitude modulation will be added to the phase coded signal. However, the PER of the commercial polarization components can be larger than $40 \mathrm{~dB}$, therefore, the effect of the PER on the phase coding performance can be neglected.

In the proposed scheme, an OF is involved. Note that the OF can be designed as a fixed spectrum characteristics filter which only removes the frequency larger than optical carrier, thus the tunability of the system can be guaranteed. On the other hand, the system stability may be suffered from the variation of the DC bias points in the modulator. This problem can be resolved by employing a DP-QPSK modulator Bias Controller (i.e. YY LABS Inc. D0158) in the system.

\section{Conclusion}

A photonics method to improve the TBWP of a linearly chirped waveform based on frequency multiplying and temporal synthesis was proposed and investigated. Firstly, the bandwidth of the linearly chirped waveform was doubled to improve the TBWP twice, then the time duration of the generated bandwidth doubled linearly chirped waveform was synthesized by $L$ times based on phase coding operation, as a result, the TBWP of the input linearly chirped waveform was increased by $2 L$ times. The generation of phase-coded frequency-doubled waveform and its TBWP improvement was theoretically analyzed and verified by simulation. In the simulation, a linearly chirped waveform with central frequency

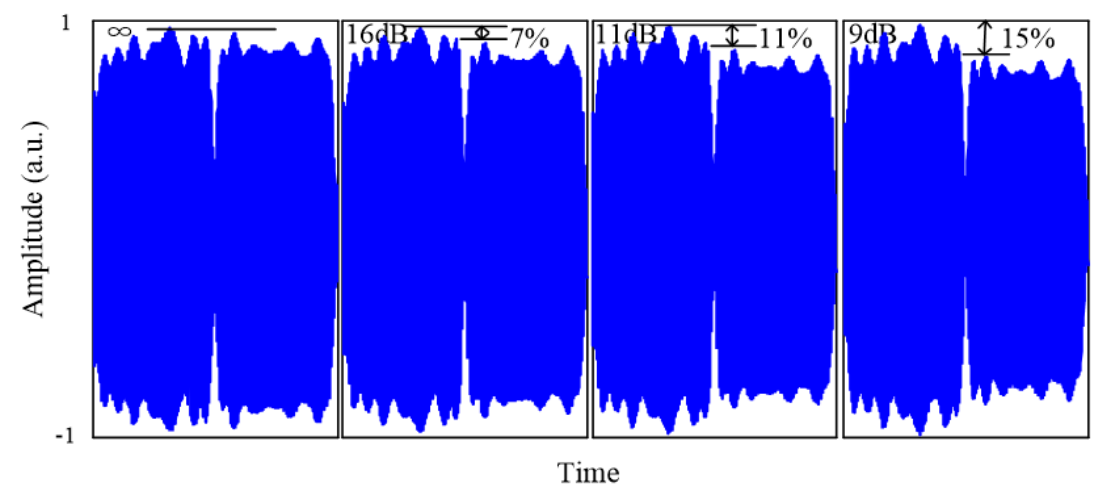

Figure 5. Effect of the finite PER on the waveform of the generated signal. 
of $8.25 \mathrm{GHz}$, bandwidth of $500 \mathrm{MHz}$, time duration of $6.4 \mathrm{~ns}$, TBWP of 3.2 was used and a synthesized waveform with central frequency of $16.5 \mathrm{GHz}$, bandwidth of $1 \mathrm{GHz}$, time duration of $819.2 \mathrm{~ns}$, TBWP of 819.2 was obtained. The proposed method features arbitrary large TBWP as well as a PN sequence with large length is utilized, and it may find applications in modern radar systems.

\section{Acknowledgements}

This work was supported by the National Natural Science Foundation of China (61901507) and the Natural Science Basis Research Plan in Shaanxi Province of China (2019JQ-707).

\section{Conflicts of Interest}

The authors declare no conflicts of interest regarding the publication of this paper.

\section{References}

[1] Richards, M.A. (2014) Fundamentals of Radar Signal Processing. Second Edition, McGraw-Hill, New York, NY, USA.

[2] Yao, J.P. (2009) Microwave Photonics. J. Lightw. Technol., 27, 314-335. https://www.osapublishing.org/jlt/abstract.cfm?URI=jlt-27-3-314 https://doi.org/10.1109/JLT.2008.2009551

[3] Minasian, R.A., Chan, E.H.W. and Yi, X. (2013) Microwave Photonics Signal Processing. J. Lightw. Technol., 31, 571-586. https://doi.org/10.1364/OE.21.022918

[4] McKinney, J., Leaird, D. and Weiner, A. (2002) Millimeter-Wave Arbitrary Waveform Generation with a Direct Space-to-Time Pulse Shaper. Optics Letters, 27, 1345-1347. https://doi.org/10.1364/OL.27.001345

[5] Maroof, H.K., Shen, H., Xuan, Y., Zhao, L., Xiao, S., Daniel, E.L., et al. (2010) Ultrabroad-Bandwidth Arbitrary Radiofrequency Waveform Generation with a Silicon Photonics Chip-Based Spectral Shaper. Nature Photonics, 4, 117-122. https://doi.org/10.1038/nphoton.2009.266

[6] Li, M. and Yao, J. (2011) Photonic Generation of Continuously Tunable Chirped Microwave Waveforms Based on a Temporal Interferometer Incorporating an Optically Pumped Linearly Chirped Fiber Bragg Grating. IEEE Transactions on Microwave Theory and Techniques, 59, 3531-3537. https://doi.org/10.1109/TMTT.2011.2169078

[7] Ashrafi, R., Park, Y. and Azaña, J. (2010) Fiber-Based Photonic Generation of High-Frequency Microwave Pulses with Reconfigurable Linear Chirp Control. IEEE Transactions on Microwave Theory and Techniques, 58, 3312-3319. https://doi.org/10.1109/TMTT.2010.2077451

[8] Zhang, Y., Ye, X., Guo, Q., Zhang, F. and Pan, S. (2017) Photonics Generation of Linear-Frequency-Modulated Waveforms with Improved Time-Bandwidth Product Based on Polarization Modulation. Journal of Lightwave Technology, 35, 1821-1829. https://doi.org/10.1109/JLT.2017.2651902

[9] Li, Y., Dezfooliyan, A. and Weiner, A.M. (2014) Photonic Synthesis of Spread Spectrum Radio Frequency Waveforms with Arbitiarily Long Time Apertures, J. Lightw. Technol., 32, 3580-3587. https://doi.org/10.1109/JLT.2014.2320933 
[10] Li, Y., Dezfooliyan, A., Wun, J.M., Leaird, D.E., Shi, J.W. and Weiner. A. M. (2014) Photonic Generation of W-Band Arbitrary Waveforms with High Time-Bandwidth Products Enabling 3.9 mm Range Resolution. Optica, 1, 446-454. https://doi.org/10.1364/OPTICA.1.000446

[11] Rashidinejad, A., Leaird, D.E. and Weiner, A.M. (2015) Ultrabroadband Radio-Frequency Arbitrary Waveform Generation with High-Speed Phase and Amplitude Modulation Capability.Opt. Exp., 23, 12265-12273. https://doi.org/10.1364/OE.23.012265

[12] Deng, H., Zhang, J., Chen, X. and Yao, J. (2017) Photonics Generation of a Phase-Coded Chirp Microwave Waveform With Increased TBWP. IEEE Photonics Technology Letters, 29, 1420-1423. https://doi.org/10.1109/LPT.2017.2717698

[13] Li, X., Zhao, S., Zhu, Z., Qu, K., Lin, T. and Hu, D. (2017) Photonic Generation of Frequency and Bandwidth Multiplying Dual-Chirp Microwave Waveform. IEEE Photonics Journal, 9,1-14. https://doi.org/10.1109/JPHOT.2017.2705042

[14] Macwilliams, F. and Sloane, N. (1976) Pseudo-Random Sequences and Arrays. Proc. IEEE, 64, 1715-1730. https://doi.org/10.1109/PROC.1976.10411

[15] Li, X., Zhao, S., Zhang, K. and Wang, G. (2020) Dual-Chirp Waveform Generation and Its TBWP Improvement Based on Polarization Modulation and Phase Coding. Optics Communications, 463, 125413. https://doi.org/10.1016/j.optcom.2020.125413 